\title{
Development and Validation of a Prognostic Model Using Blood Biomarker Information for Prediction of Survival of Non-Small-Cell Lung Cancer Patients Treated With Combined Chemotherapy and Radiation or Radiotherapy Alone (NCT00181519, NCT00573040, and NCT00572325).
}

Citation for published version (APA):

Dehing-Oberije, C. J. G., Aerts, H., Yu, S., de Ruysscher, D., Menheere, P. P. C. A., Hilvo, M., van der Weide, H., Rao, B., \& Lambin, P. (2011). Development and Validation of a Prognostic Model Using Blood Biomarker Information for Prediction of Survival of Non-Small-Cell Lung Cancer Patients Treated With Combined Chemotherapy and Radiation or Radiotherapy Alone (NCT00181519, NCT00573040, and NCT00572325). International Journal of Radiation Oncology Biology Physics, 81(2), 360-368. https://doi.org/10.1016/j.ijrobp.2010.06.011

Document status and date:

Published: 01/10/2011

DOI:

10.1016/j.ijrobp.2010.06.011

Document Version:

Publisher's PDF, also known as Version of record

Document license:

Taverne

Please check the document version of this publication:

- A submitted manuscript is the version of the article upon submission and before peer-review. There can be important differences between the submitted version and the official published version of record.

People interested in the research are advised to contact the author for the final version of the publication, or visit the DOI to the publisher's website.

- The final author version and the galley proof are versions of the publication after peer review.

- The final published version features the final layout of the paper including the volume, issue and page numbers.

Link to publication

\footnotetext{
General rights rights.

- You may freely distribute the URL identifying the publication in the public portal. please follow below link for the End User Agreement:

www.umlib.nl/taverne-license

Take down policy

If you believe that this document breaches copyright please contact us at:

repository@maastrichtuniversity.nl

providing details and we will investigate your claim.
}

Copyright and moral rights for the publications made accessible in the public portal are retained by the authors and/or other copyright owners and it is a condition of accessing publications that users recognise and abide by the legal requirements associated with these

- Users may download and print one copy of any publication from the public portal for the purpose of private study or research. - You may not further distribute the material or use it for any profit-making activity or commercial gain

If the publication is distributed under the terms of Article 25fa of the Dutch Copyright Act, indicated by the "Taverne" license above, 


\title{
DEVELOPMENT AND VALIDATION OF A PROGNOSTIC MODEL USING BLOOD BIOMARKER INFORMATION FOR PREDICTION OF SURVIVAL OF NON-SMALL-CELL LUNG CANCER PATIENTS TREATED WITH COMBINED CHEMOTHERAPY AND RADIATION OR RADIOTHERAPY ALONE (NCT00181519, NCT00573040, AND NCT00572325)
}

\author{
Cary Dehing-Oberije, M.Sc., ${ }^{*}$ Hugo Aerts, M.Sc., ${ }^{*}$ Shipeng Yu, Ph.D., ${ }^{\dagger}$ \\ Dirk De Ruysscher, M.D., Ph.D., ${ }^{*}$ Paul Menheere, Ph.D., ${ }^{\ddagger}$ Mika Hilvo, Ph.D., ${ }^{\Uparrow}$ \\ Hiska van der Weide, M.D., ${ }^{*}$ Bharat Rao, Ph.D., ${ }^{\dagger}$ and Philippe Lambin, M.D., Ph.D.* \\ *Department of Radiation Oncology (MAASTRO Clinic), GROW-School for Oncology and Developmental Biology, and ${ }^{\ddagger}$ Department \\ of Clinical Chemistry, Maastricht University Medical Centre, Maastricht, The Netherlands; ${ }^{\dagger}$ Siemens Medical Solutions, Malvern, PA; \\ ${ }^{\S}$ Institute of Medical Technology, University of Tampere, Tampere, Finland; and ${ }^{\|}$VTT Technical Research Centre of Finland, Espoo, Finland
}

\begin{abstract}
Purpose: Currently, prediction of survival for non-small-cell lung cancer patients treated with (chemo)radiotherapy is mainly based on clinical factors. The hypothesis of this prospective study was that blood biomarkers related to hypoxia, inflammation, and tumor load would have an added prognostic value for predicting survival.

Methods and Materials: Clinical data and blood samples were collected prospectively (NCT00181519, NCT00573040, and NCT00572325) from 106 inoperable non-small-cell lung cancer patients (Stages I-IIIB), treated with curative intent with radiotherapy alone or combined with chemotherapy. Blood biomarkers, including lactate dehydrogenase, C-reactive protein, osteopontin, carbonic anhydrase IX, interleukin (IL) 6, IL-8, carcinoembryonic antigen (CEA), and cytokeratin fragment 21-1, were measured. A multivariate model, built on a large patient population $(N=322)$ and externally validated, was used as a baseline model. An extended model was created by selecting additional biomarkers. The model's performance was expressed as the area under the curve (AUC) of the receiver operating characteristic and assessed by use of leave-one-out cross validation as well as a validation cohort $(n=52)$.

Results: The baseline model consisted of gender, World Health Organization performance status, forced expiratory volume, number of positive lymph node stations, and gross tumor volume and yielded an AUC of 0.72. The extended model included two additional blood biomarkers (CEA and IL-6) and resulted in a leave-one-out AUC of 0.81 . The performance of the extended model was significantly better than the clinical model $(p=0.004)$. The AUC on the validation cohort was 0.66 and 0.76 , respectively.

Conclusions: The performance of the prognostic model for survival improved markedly by adding two blood biomarkers: CEA and IL-6. (c) 2011 Elsevier Inc.
\end{abstract}

NSCLC, Prognostic model, Radiotherapy, Chemotherapy, Blood biomarker, Prognosis.

\section{INTRODUCTION}

Many lung cancer patients are treated with radiotherapy (RT) alone or RT combined with chemotherapy (1). Although an accurate assessment of prognosis is of the utmost importance for patients as well as for clinicians, it is widely known that the TNM staging system is suboptimal to achieve this goal (2-4). To improve risk stratification, a number of variables associated with survival have been identified. At present, the generally accepted prognostic factors for survival of inoperable patients are performance status $(5,6)$, weight loss $(5,6)$, presence of comorbidity (7), use of chemotherapy in addition to RT $(6,8)$, and tumor size $(2-4,9-11)$. Although there are no randomized data available, retrospective studies suggest that a higher radiation dose leads to improved local control and better survival rates $(5,6,12)$. For other factors, such as gender and age, the literature shows
Reprint requests to: Cary Dehing-Oberije, M.Sc., MAASTRO Clinic, Dr. Tanslaan 12, 6229 ET Maastricht, The Netherlands. Tel: +31 88 4455666; Fax: +31 88 4455667; E-mail: cary. dehing@maastro.nl

This research was partially supported by a grant from the DeZnIT project of the European Union's Sixth Framework Programme, as well as the AIRFORCE project (Grant 03O-103), and was per- formed within the framework of the Center for Translational Molecular Medicine.

Conflict of interest: S.Y. and B.R. are employed by the Image and Knowledge Management division of Siemens Medical Solutions. No actual or potential conflicts of interest exist for the other coauthors.

Received March 29, 2010, and in revised form May 26, 2010. Accepted for publication June 8, 2010. 
inconsistent results, making it difficult to draw definitive conclusions (5).

A prognostic model for the survival of non-surgically treated patients was recently published, consisting of five variables: gender, performance status, forced expiratory volume in 1 second $\left(\mathrm{FEV}_{1}\right)$, number of positive lymph node stations (PLNS), and gross tumor volume (GTV) (13). The model was validated on two external data sets, resulting in an area under the curve (AUC) of the receiver operating characteristic (ROC) of 0.75 and 0.76. Although these results are promising, there is still a great deal of room for improvement in survival prediction. For a subgroup of 106 patients, we had blood samples available to refine and improve the model.

The analysis of circulating proteins can provide useful additional information about the biological characteristics of tumors (14) and their hosts. Blood obtained by venal puncture is an easily accessible human specimen and can be monitored over long periods. Moreover, the value of blood biomarkers and imaging parameters in these patients is emphasized by the fact that extensive tumor tissue sampling is very difficult in many cases of lung cancer.

In recent decades, research has shown a number of biomarkers to be associated with survival or disease progression, mostly in univariate analyses (15-21) but also in multivariate analyses (21-24). However, their ability to improve the survival prediction in terms of higher AUC values has not yet been examined. In this study we chose eight blood biomarkers. Osteopontin (OPN), carbonic anhydrase IX (CA IX), and lactate dehydrogenase (LDH) are hypoxia markers; expression of carcinoembryonic antigen (CEA) and cytokeratin fragment (CYFRA) 21-1 is correlated with tumor load; and interleukin (IL) 6, IL-8, and C-reactive protein (CRP) are related to inflammation. In previous studies these blood biomarkers were found to be statistically significant factors for survival of lung cancer patients (15-24).

We carried out this prospective study to assess the prognostic value of these 8 blood biomarkers as well as test the hypothesis that these blood biomarkers improve the prediction of 2-year survival of non-small-cell lung cancer (NSCLC) patients treated with RT alone or RT combined with chemotherapy compared with a model consisting of clinical factors only.

\section{METHODS AND MATERIALS}

\section{Patient population}

Between May 2004 and November 2007, 339 nonsurgical Stage I-IIIB NSCLC patients were referred to the MAASTRO Clinic, Maastricht, the Netherlands, to be treated with curative intent. Data and blood samples were collected prospectively, ensuring standardization of data collection. Blood samples were available from 158 NSCLC patients, who were willing to participate in the Biobank lung cancer study (NCT00573040). A split-sample validation was performed with a typical split of $2 / 3: 1 / 3$. Thus the model was built on two-thirds of the data (106 patients included between May 2004 and May 2006) and validated on the remaining one-third (52 patients included between June 2006 and
November 2007). The primary GTV and nodal GTV were calculated based on the pretreatment ${ }^{18} \mathrm{~F}$-deoxyglucose positron emission tomography and computed tomography scans, by use of an RT treatment-planning system (Computerized Medical Systems, St. Louis, MO) (25). The sum of primary GTV and nodal GTV resulted in the GTV. Stage I-II patients did not have invasive mediastinal staging, but they had no mediastinal involvement, based on a negative computed tomography and ${ }^{18} \mathrm{~F}$-deoxyglucose-positron emission tomography scan.

In patients with $\mathrm{T} 1-\mathrm{T} 3$ tumors, the $\mathrm{N} 2$ or $\mathrm{N} 3$ status was confirmed by endoscopic ultrasound, endobronchial ultrasound, and ultrasound-guided fine-need aspiration in case of supraclavicular invasion or by mediastinoscopy. Patients with radiologic T4 tumors did not have their lymph node status pathologically confirmed.

For patients treated with sequential chemotherapy, stage and PLNS were assessed by use of pre-chemotherapy imaging information.

\section{Blood samples}

All patients participated in a prospective study (NCT00181519), launched at the MAASTRO Clinic in 2003 (26). Before the start of the RT treatment, blood samples were collected, processed, and stored in a standardized manner. The plasma or serum level of the investigated proteins was determined in all specimens by use of commercially available kits in line with the manufacturers' instructions. All samples were analyzed simultaneously. Measurements were performed for biomarkers related to (1) hypoxia-LDH (Beckman Coulter, Fullerton, CA), CA IX (MN/CA IX ELISA; Siemens Medical Solutions Diagnostics, Tarrytown, NY), and OPN (Quantikine Human Osteopontin Immuno assay; R\&D Systems, Minneapolis, MN); (2) inflammation-IL-6 (Siemens Medical Solutions Diagnostics), IL-8 (Siemens Medical Solutions Diagnostics), and CRP (Beckman Coulter); and (3) tumor load-CYFRA 21-1 (Brahms Aktiengesellschaft, Hennigsdorf, Germany), CEA (Siemens Medical Solutions Diagnostics), and OPN. We determined OPN and CA IX by manual methods, and these were therefore measured in duplicate; the others were measured once.

\section{$R T$ treatment}

Patients were treated in accordance with different radiation and chemotherapy protocols defined a priori according to the stage of the disease. No elective nodal irradiation was performed (27), and irradiation was delivered 5 days per week. Radiotherapy planning was performed with an $\mathrm{XiO}$ system (Computerized Medical Systems), by use of a convolution-superposition algorithm with inhomogeneity corrections and according to International Commission on Radiation Units \& Measurements 50 guidelines. Three different RT regimens were administered:

1. Forty-eight patients were treated according to the standard protocol used until August 2005. They received either 70 Gy (Stage I-II) or 60 Gy after induction chemotherapy (Stage III) in once-daily fractions of $2 \mathrm{~Gy}$. The induction chemotherapy consisted of carboplatin on Day 1 and gemcitabine on Days 1 and 8 . The majority of the patients received 3 cycles (range, 1-6).

2. Ninety-five patients were treated according to the new protocol for sequential chemotherapy and radiation (Stage III) or RT alone (Stage I-II), which was introduced in August 2005 (28, 29). The individualized radiation dose ranged from 54.0 to $79.2 \mathrm{~Gy}$, delivered in fractions of 1.8 Gy twice daily, depending on the mean lung dose or the spinal cord dose constraint. An 8-h 
interval between the fractions was respected. The same induction chemotherapy regimen was applied as in Group 1.

3. Fifteen patients received concurrent chemoradiotherapy (30). After 2 cycles of carboplatin-gemcitabine, a radiation dose of 45 Gy was delivered in fractions of 1.5 Gy twice daily, followed by an individualized dose ranging from 8 to $24 \mathrm{~Gy}$, depending on the mean lung dose or the spinal cord dose constraint, delivered in fractions of $2.0 \mathrm{~Gy}$ once daily. Cisplatin-vinorelbine was given concurrently on Days 2, 9, 23, and 30.

\section{Statistical analysis}

Overall survival was defined as the duration between the start of RT and the date of death. Survival status was evaluated in November 2009. Data were considered right-censored if patients were alive at the time of evaluation.

The statistical significance of the differences between the development and validation cohorts were assessed with the $t$ test or chi-square test. Because the biomarker levels showed a skewed distribution, the Mann-Whitney $U$ test was applied for these variables. The Kaplan-Meier method was used for univariate survival analysis. Blood biomarker concentrations were dichotomized by use of the median value as a cutoff point. The log-rank test was applied to assess differences between groups.

By use of 2-norm support vector machines (31), a multivariate prediction model for 2-year survival was built based on data from the development cohort $(n=106)$. Missing values were imputed by use of single imputation. A logarithmic transformation was applied to obtain more symmetrically distributed data for the blood biomarkers.

A multivariate model, built on a large patient population and externally validated, was used as a baseline model. Details of this model as well as a description of the patient population have been published previously (13). In short, it consisted of five variables: gender, World Health Organization performance status (WHOPS), $\mathrm{FEV}_{1}$, PLNS, and GTV. To assess the added prognostic value of the blood biomarkers, the baseline model was extended with the biomarkers mentioned previously. A combinatorial variable selection procedure was performed to obtain the "best subset" of blood biomarker variables. The best subset was defined as the combination of biomarkers that resulted in the highest AUC of the receiver operating curve (ROC), assessed by leave-one-out (LOO) cross validation. The maximum value of the AUC is 1.0, indicating a perfect prediction model. A value of 0.5 indicates that patients are correctly classified in $50 \%$ of the cases (i.e., as good as chance).

The coefficients and confidence intervals (CIs) of the model were calculated by use of a bootstrap procedure, repeated 1000 times. The coefficients were then multiplied by a correction factor to obtain odds ratios (32). In addition, a nomogram, which is a graphical representation of a multivariate model, was created for the combined model. Each effect in the model is converted to a 0 to 100 scale, which is just proportional to the log odds. These points are added across predictors to derive the "total points," which are converted to a predicted probability.

The validation cohort was split into three subgroups based on tertiles of the predicted probability, which can be calculated by use of the coefficients of the multivariate model. To assess differences in survival of the subgroups, Kaplan-Meier curves were made. The risk stratification based on the extended model was compared with the risk stratification based on clinical TNM stage (sixth edition). To quantify the improvement in performance of adding biomarker information, the AUC of the baseline as well as the combined model was calculated. The statistical significance of the differences was assessed by a bootstrap technique. The analysis was performed with R, version 2.8.1 (R Foundation for Statistical Computing, Vienna, Austria) and Matlab, version 2008b (The MathWorks, Natick, MA).

\section{Ethics}

This study was conducted according to national laws and guidelines and approved by the appropriate local trial committee. Informed consent was obtained from all participants.

\section{RESULTS}

The patient characteristics of the development as well as the validation cohort are shown in Table 1 . The median follow-up of the development cohort was 38 months (range, 1-71 months); the median survival, 15 months (95\% CI, 1317 months); and the 2-year survival, 33\% (95\% CI, 24-42\%). Two years after the RT treatment, 35 patients were alive whereas 71 had died. At the time of the analysis (November 2009), 14 patients were still alive. The results of the biomarker measurements are shown in Table 2. The log-rank test showed statistically significant results for CEA, IL-6, OPN, and CYFRA 21-1 ( $p=0.013, p=0.003, p=0.004$, and $p<0.001$, respectively). Higher biomarker levels were associated with a lower probability of survival. With the exception of CA IX, the same trend was observed for the other biomarkers-CRP, LDH, and IL-8 - but the log-rank test did not yield statistically significant results. Kaplan-Meier survival curves are shown in Fig. 1.

\section{Multivariate model}

The baseline model consisted of five variables: gender, WHO-PS, FEV 1 , GTV, and PLNS. Details of this model as well as a description of the patient population have been published elsewhere (13). In the new, extended model with biomarker information, we included CEA, CRP, CA IX, OPN, CYFRA 21-1, LDH, IL-6, and IL-8. After the variable selection procedure, the final model consisted of all five variables of the baseline model and two additional biomarkers: CEA and IL-6. The most powerful prognostic factors for 2-year survival were GTV and CEA. The AUC of the final model was 0.81 (95\% CI, 0.74-0.92), assessed by LOO cross validation, whereas the baseline model, applied to the development cohort of 106 patients, yielded an AUC of $0.72(95 \%$ CI, 0.62-0.84). The ROC curves are shown in Fig. 2A. The difference between the two models was statistically significant $(p=0.004)$. The odds ratios for the variables are shown in Table 3. The coefficients of the extended model were used to create a nomogram (Fig. 3).

\section{Validation cohort}

The median follow-up of the validation cohort was 30 months (range, 2-41 months), whereas the median survival was 21 months (95\% CI, 11-31 months) and the 2-year survival was $48 \%$ (95\% CI, 35-62\%). In this validation cohort the AUC of the baseline model was 0.66 whereas the extended model yielded an AUC of 0.76 (Fig. 2B). To create 
Table 1. Patient characteristics

\begin{tabular}{|c|c|c|c|}
\hline & Development cohort $(n=106)$ & Validation cohort $(n=52)$ & $p$ Value \\
\hline Gender & & & 0.075 \\
\hline Male & $85(80.2 \%)$ & $35(67.3 \%)$ & \\
\hline Female & $21(19.8 \%)$ & $17(32.7 \%)$ & \\
\hline Age (y) & Mean, $68.5(\mathrm{SD}, 9.9)$ & Mean, $66.2(\mathrm{SD}, 11.7)$ & 0.190 \\
\hline WHO-PS & & & 0.888 \\
\hline 0 & $34(32.7 \%)$ & $15(28.8 \%)$ & \\
\hline 1 & $55(52.9 \%)$ & $29(55.8 \%)$ & \\
\hline$\geq 2$ & $15(14.4 \%)$ & $8(15.4 \%)$ & \\
\hline $\mathrm{FEV}_{1}(\%)$ & Mean, $71.6(\mathrm{SD}, 23.8)$ & Mean, $72.5(\mathrm{SD}, 19.8)$ & 0.820 \\
\hline Histology & & & 0.009 \\
\hline $\mathrm{SCC}$ & $38(35.8 \%)$ & $8(15.4 \%)$ & \\
\hline Adenocarcinoma & $16(15.1 \%)$ & $7(13.5 \%)$ & \\
\hline Large cell carcinoma & $33(31.2 \%)$ & $18(34.6 \%)$ & \\
\hline Other & $9(8.5 \%)$ & $14(26.9 \%)$ & \\
\hline No histology & $10(9.4 \%)$ & $5(9.6 \%)$ & \\
\hline Clinical stage & & & 0.781 \\
\hline $\mathrm{I}$ & $18(17.0 \%)$ & $10(19.2 \%)$ & \\
\hline II & $10(9.4 \%)$ & $4(7.7 \%)$ & \\
\hline IIIA & $24(22.6 \%)$ & $15(28.9 \%)$ & \\
\hline IIIB & $54(51.0 \%)$ & $23(44.2 \%)$ & \\
\hline Gross tumor volume (mL) & Mean, 105 (SD, 99) & Mean, $103(\mathrm{SD}, 131)$ & 0.106 \\
\hline PLNS & & & 0.985 \\
\hline 0 & $33(34.0 \%)$ & $17(35.4 \%)$ & \\
\hline 1 & $21(21.6 \%)$ & $10(20.8 \%)$ & \\
\hline$\geq 2$ & $43(44.4 \%)$ & $21(43.8 \%)$ & \\
\hline Chemotherapy & & & 0.001 \\
\hline No & $34(32.1 \%)$ & $16(30.8 \%)$ & \\
\hline Sequential & $69(65.1 \%)$ & $25(48.1 \%)$ & \\
\hline Concurrent & $3(2.8 \%)$ & $11(21.1 \%)$ & \\
\hline $\mathrm{EQD}_{2 \mathrm{~T}}(\mathrm{~Gy})$ & Mean, 56.7 (SD, 8.9) & Mean, $59.5(\mathrm{SD}, 9.5)$ & 0.074 \\
\hline OTT (d) & Mean, 33 (SD, 10) & Mean, 27 (SD, 7) & $<0.001$ \\
\hline
\end{tabular}

Abbreviations: WHO-PS = World Health Organization performance status; $\mathrm{FEV}_{1}=$ forced expiratory volume in 1 second; $\mathrm{SCC}=$ squamous

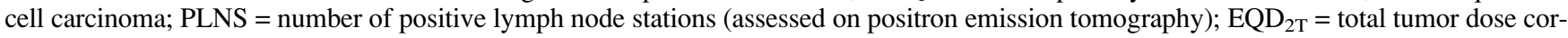
rected for fraction size and overall treatment time; OTT $=$ overall treatment time.

low-, medium-, and high-risk groups, the cohort was split into three subgroups, based on tertiles of the predicted probability of the extended model. In addition, the overall TNM stage (sixth edition) was used as risk stratification. The Kaplan-
Meier survival curves are shown in Fig. 4. The 2-year survival of the low-, medium-, and high-risk groups was $77 \%$ (95\% CI, 56-97\%), 39\% (95\% CI, 16-62\%), and 29\% (95\% CI, 7$51 \%$ ), respectively, and the log-rank test yielded a statistically

Table 2. Biomarkers in blood

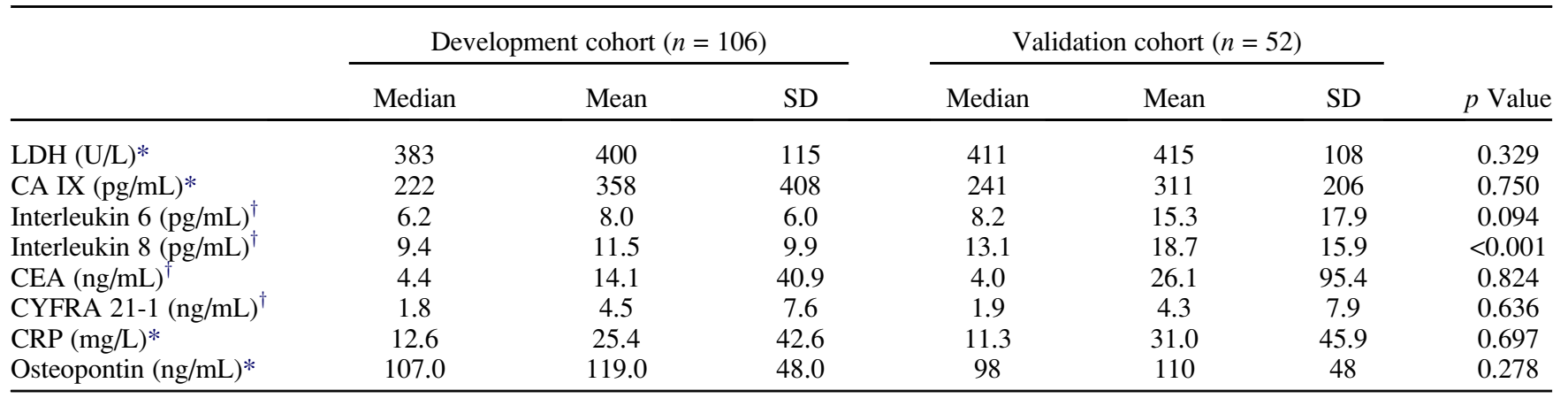

Abbreviations: $\mathrm{LDH}=$ lactate dehydrogenase; CA IX = carbonic anhydrase IX; CEA = carcinoembryonic antigen; CYFRA 21-1 = cytokeratin fragment 21-1; CRP = C-reactive protein.

* Measurements performed by use of plasma.

${ }^{\dagger}$ Measurements performed by use of serum. 
A.

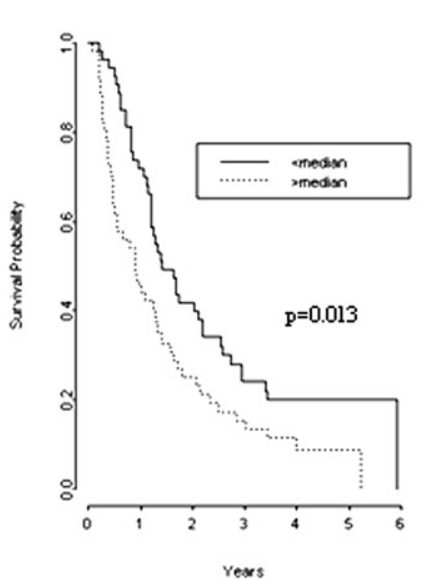

E. Carboric Armycuase ix

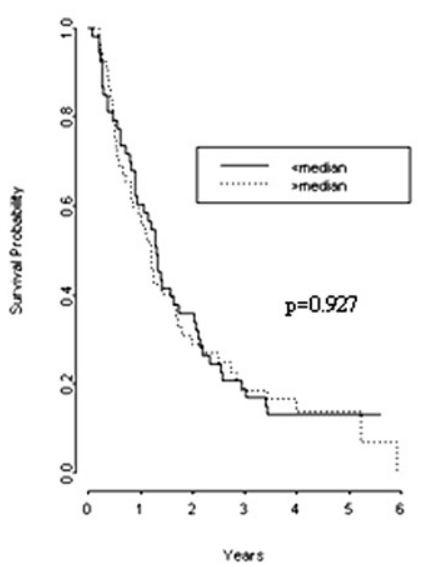

B.

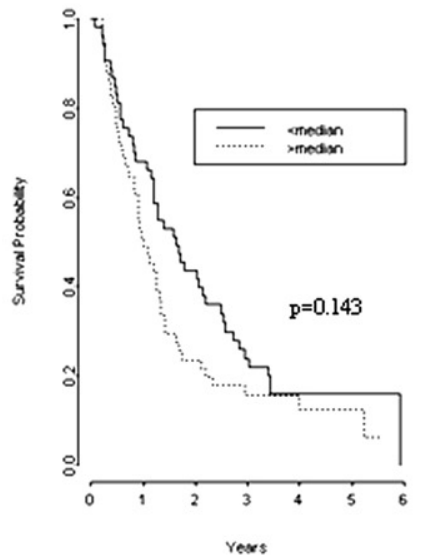

F.

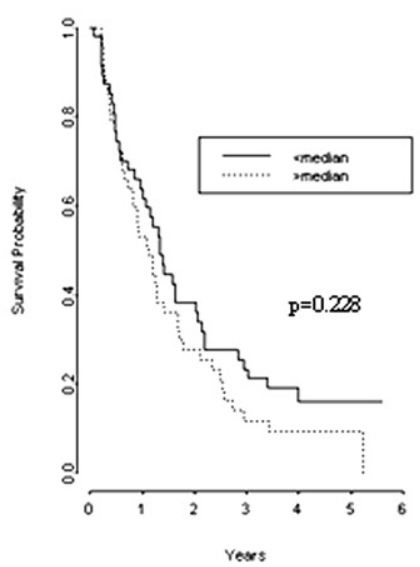

C.

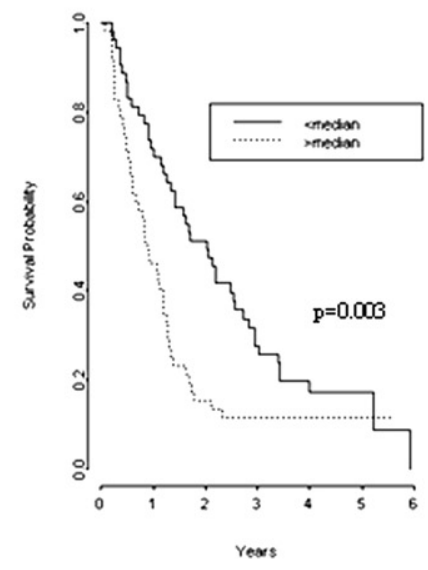

G. cytro21-1

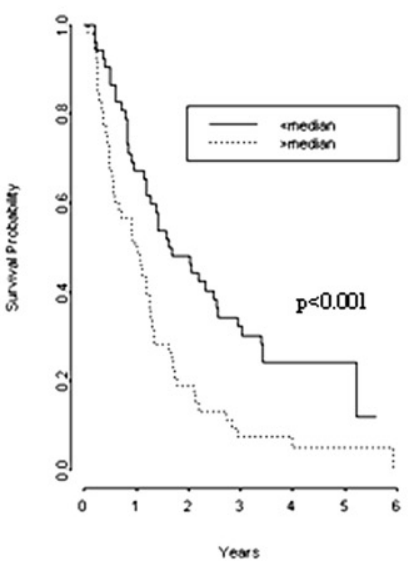

D. Ostecporten

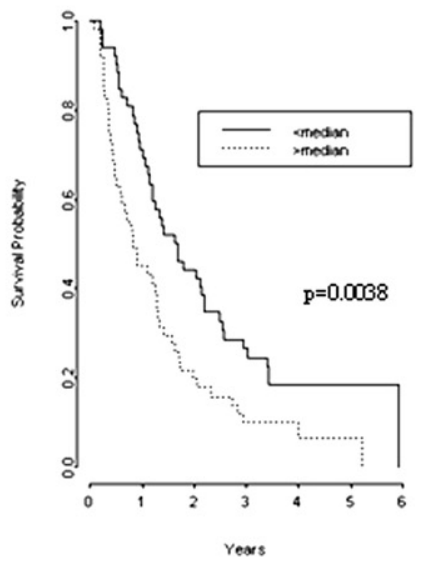

H. crextrue pectern

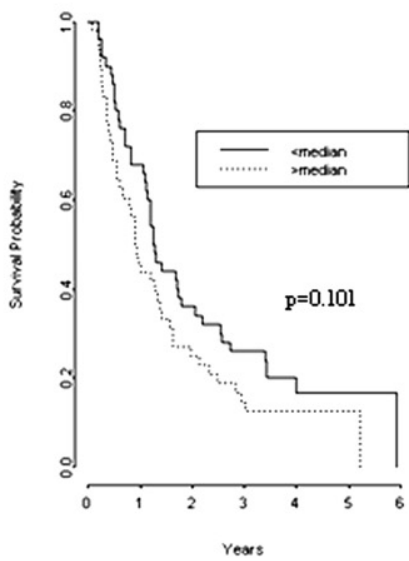

Fig. 1. Kaplan-Meier curves for overall survival according to biomarker concentration in blood of (A) interleukin 6, (B) interleukin 8, (C) carcinoembryonic antigen, (D) osteopontin, (E) carbonic anhydrase IX, (F) lactate dehydrogenase, (G) cytokeratin fragment 21-1, and (H) C-reactive protein ( $n=106$, development cohort).

significant $p$ value of 0.002 . According to this risk stratification, 4 patients (27\%) with Clinical Stage IIIA and 5 patients (22\%) with Clinical Stage IIIB were included in the low-risk group. The risk stratification based on overall TNM stage re-

(a)

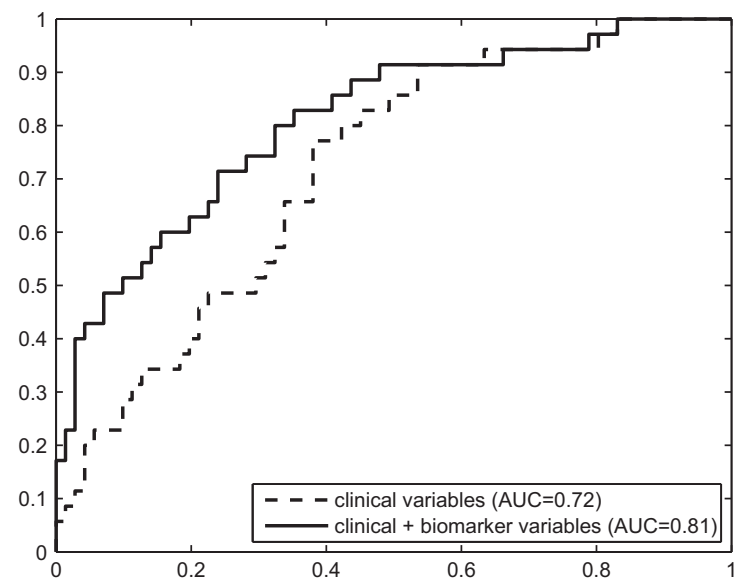

sulted in a 2-year survival of $43 \%$ (95\% CI, 17-69\%) for Stage I-II, 53\% (95 CI, 28-66\%) for Stage IIIA, and 48\% (95\% CI, $27-68 \%)$ for Stage IIIB patients. The log-rank test for overall TNM stage was not statistically significant $(p=0.89)$.

(b)

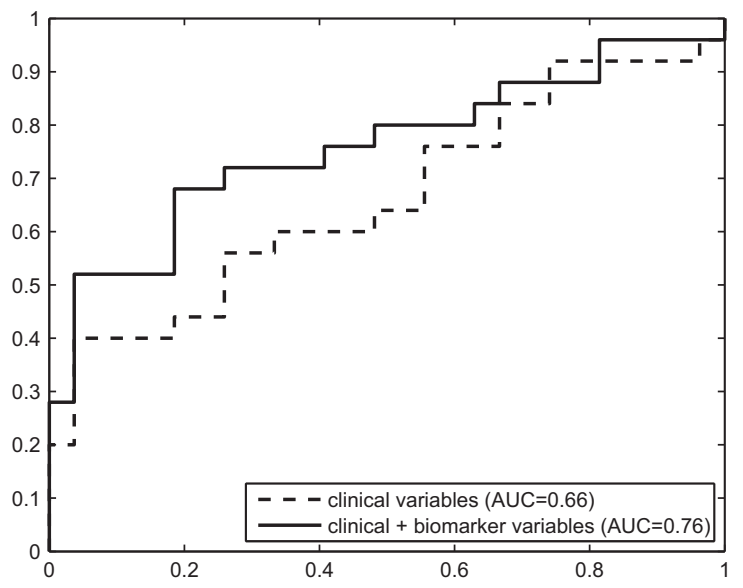

Fig. 2. Receiver operating characteristic (ROC) curves for (A) the development cohort, which consisted of 106 patients, and (B) the validation cohort, which consisted of 52 patients. The extended model (clinical + biomarker variables) performs better in both cohorts. AUC $=$ area under the curve. 
Table 3. Odds ratios for being alive at 2-year time point

\begin{tabular}{|c|c|c|c|c|}
\hline Variable & Coefficient & Odds ratio & $95 \% \mathrm{CI}$ & $p$ Value \\
\hline Gender & & & & 0.770 \\
\hline Male & & Reference & & \\
\hline Female & 0.21 & 1.24 & $0.30-5.14$ & \\
\hline WHO-PS & & & & 0.037 \\
\hline 0 & & Reference & & \\
\hline 1 & -1.01 & 0.36 & $0.14-0.94$ & \\
\hline$\geq 2$ & -2.03 & 0.13 & $0.05-0.34$ & \\
\hline $\mathrm{FEV}_{1}$ & -0.01 & 0.99 & $0.96-1.02$ & 0.491 \\
\hline PLNS & & & & 0.530 \\
\hline 0 & & Reference & & \\
\hline 1 & -0.14 & 0.87 & $0.57-1.33$ & \\
\hline 2 & -0.27 & 0.76 & $0.50-1.16$ & \\
\hline 3 & -0.41 & 0.66 & $0.43-1.02$ & \\
\hline$\geq 4$ & -0.54 & 0.58 & $0.38-0.89$ & \\
\hline $\ln (\mathrm{GTV})(\mathrm{mL})$ & -1.13 & 0.32 & $0.18-0.59$ & $<0.001$ \\
\hline CEA* & -2.57 & 0.08 & $0.02-0.25$ & $<0.001$ \\
\hline IL-6* & -2.43 & 0.09 & $0.01-1.02$ & 0.051 \\
\hline
\end{tabular}

Abbreviations: $\mathrm{CI}=$ confidence interval; WHO-PS $=$ World Health Organization performance status; $\mathrm{FEV}_{1}=$ forced expiratory volume in 1 second; PLNS = number of positive lymph node stations; $\ln =$ natural logarithm; GTV = gross tumor volume; $\mathrm{CEA}=$ carcinoembryonic antigen; IL-6 = interleukin 6 .

* Logarithmic transformation was used for analysis.

\section{DISCUSSION}

In this study we were able to improve the performance of a prognostic model for 2-year survival of NSCLC patients treated with (chemo)radiotherapy by incorporating biomarker information. The performance of the model increased from 0.72 to 0.81 , which was a highly statistically significant improvement $(p=0.004)$. From a panel of eight biomarkers, CEA and IL-6 were selected for inclusion in the multivariate model.

CEA serum levels are a well-established prognostic factor in colorectal, breast, and lung cancer (33). As the tumor size increases, more CEA accumulates in the blood. Patients with an increased CEA level have both a shorter disease-free survival and a lower overall survival than those with normal CEA levels (34). In a study that included 300 patients treated with RT, CEA was found to be statistically significant in univariate survival analysis (35). Our findings are in line with these results.

Although inflammation may sometimes slow the cancer, there is increasing evidence suggesting that cancer can take advantage of inflammatory responses, and the inflammatory cytokines released by the immune reaction may in fact fuel cancer progression (36-38). We found that a higher level

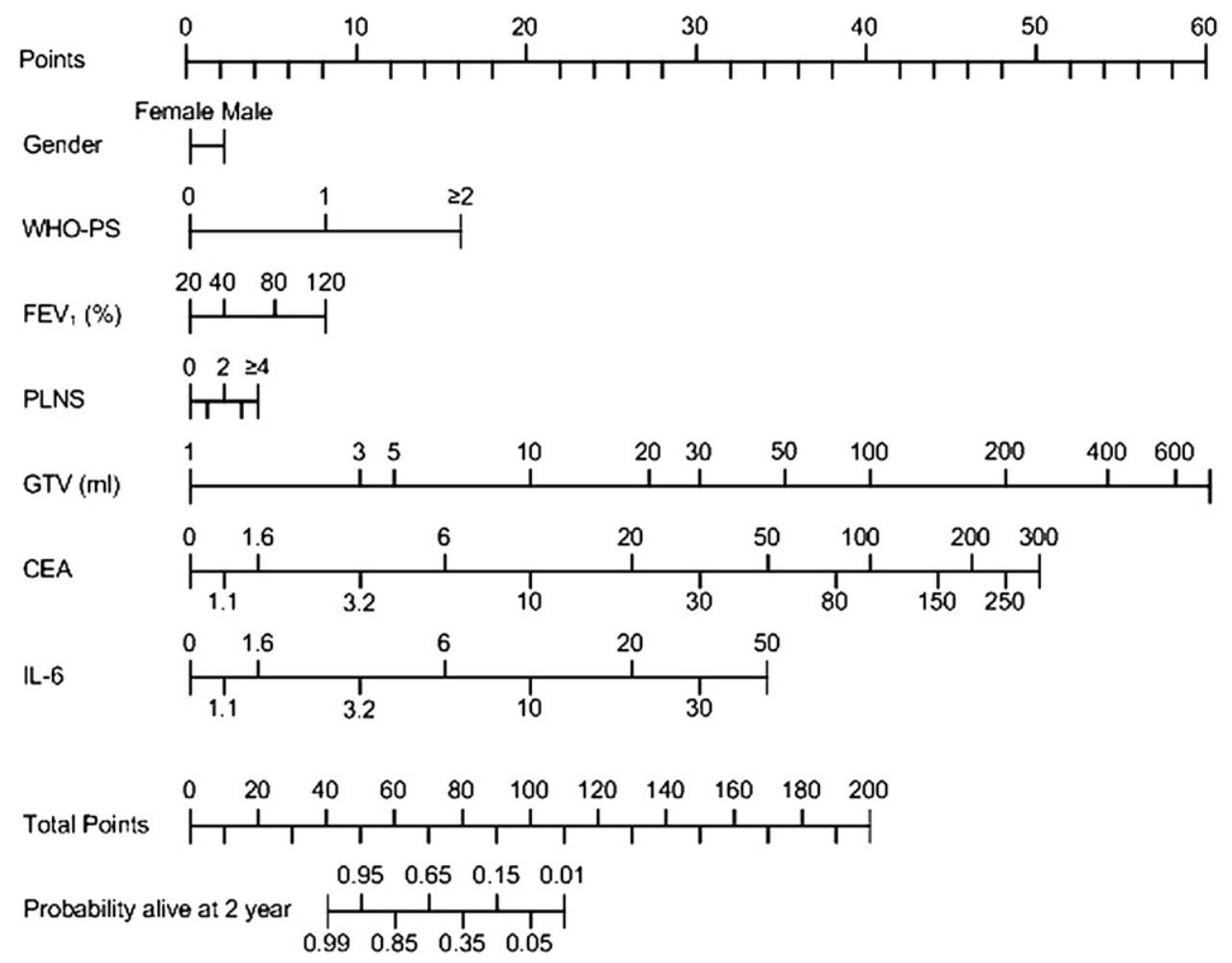

Fig. 3. Nomogram for prediction of 2-year survival. The instructions for use are as follows: Locate the patient's gender on the gender axis. Draw a line straight upward to the points axis to determine how many points a patients receives for age. Repeat this process for the other axes, each time drawing straight upward to the points axis. Sum the points achieved for each predictor and locate this sum on the "total points" axis. Draw a line straight down to assess the probability for this patient. WHO-PS = World Health Organization performance status; $\mathrm{FEV}_{1}=$ forced expiratory volume in 1 second; PLNS = number of positive lymph node stations; GTV = gross tumor volume; CEA = carcinoembryonic antigen; IL- $6=$ interleukin 6 . 

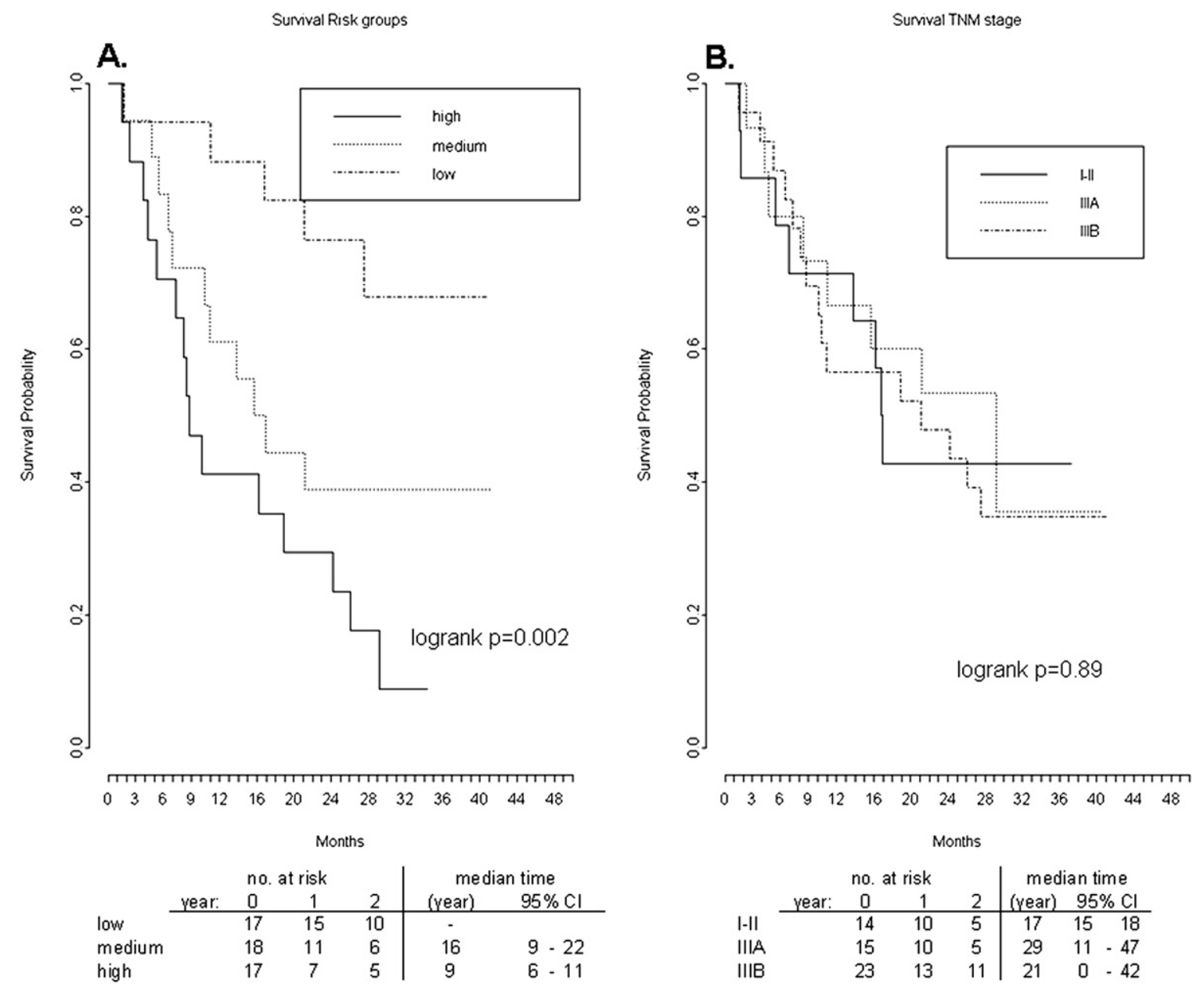

Fig. 4. Kaplan-Meier Curves for overall survival according to (A) risk group and (B) TNM stage $(n=52$, validation cohort). $\mathrm{CI}=$ confidence interval.

of IL-6 was indicative of a lower probability of survival. However, higher blood levels of inflammatory markers have also been associated with lower survival in patients with chronic obstructive pulmonary disease and cardiovascular diseases (39). Because these are very common comorbidities in lung cancer patients, this might offer an alternative explanation for our finding.

To test our hypothesis that blood biomarker information improves the prediction of 2-year survival of NSCLC patients compared with a model consisting of clinical factors only, we measured eight blood biomarkers related to hypoxia, inflammation, and tumor load. Although the selected biomarkers have already been associated with either survival or tumor control in previous studies (15-24) and all could be linked to biological pathways, it is likely that other blood biomarkers also might have a comparable prognostic value.

Biomarker measurements were performed on pretreatment blood samples. Given the complex interplay between tumor processes such as hypoxia, inflammation, and acidosis; treatment characteristics; and the expression of biomarkers, future studies should incorporate measurements at several time points to monitor biomarker levels and investigate the prognostic meaning of therapy-induced changes.
Gene array analysis might result in extra prognostic information. However, to perform a reliable analysis thorough and, representative sampling of different tumor areas is required. This can seldom be done in inoperable NSCLC patients. Therefore, at the moment, the applicability of gene array analysis for prediction of prognosis in these NSCLC patients is very limited.

Although the selection procedure for the baseline model included a number of treatment characteristics such as total treatment dose, overall treatment time, equivalent radiation dose corrected for fraction size and time, and chemotherapy, their association with survival was not strong enough to be ultimately selected in the model. This contradicts clinical trials that have reported a statistically significant influence of treatment parameters [e.g., sequential chemotherapy (8) and overall treatment time (40)] on survival rate. However, these effects are often small, and the results are usually obtained by use of a highly selected and rather homogeneous study population. In the general patient population, which is quite heterogeneous, patient as well as tumor characteristics are often more important prognostic factors than treatment characteristics. It is widely known that replicating trial results in the general patient population is often difficult. 
In this study a limited number of patients were included. To avoid overfitting, the model's performance was assessed by use of LOO cross validation. In addition, variable selection was only performed for the biomarker variables, whereas a baseline model built on a large patient population was used to determine which clinical variables to include in the multivariate model.

The model was validated in a new patient cohort. Although the AUCs slightly decreased in the validation cohort, the difference between the baseline model and the extended model remained the same, indicating that the added prognostic value of the selected biomarkers did not change.

According to our model, some patients with Clinical Stage IIIA or IIIB were classified in the low-risk group, emphasizing the extreme heterogeneity within the TNM staging groups. However, recently, the seventh edition of the TNM staging system for lung cancer was introduced (41), but for our study population, the sixth edition was used. Although it seems unlikely that a major shift would occur in the stage grouping, we cannot exclude the possibility that application of the seventh edition of the TNM staging system would result in an improved risk stratification.

Currently, clinical guidelines are based on stage, performance status, and weight loss, ignoring information about tumor volume, positive lymph node stations, and biomarkers. Our model shows that other identifiable factors play an important role in the heterogeneous outcome of patients. Further refinement will lead to more individualized treatment approaches.

\section{CONCLUSIONS}

Our results show that more accurate survival prediction is possible. In the near future, research should focus on identifying subgroups of patients who might benefit most from this additional information, thereby improving the treatment decision-making process for these patients as well as restricting the extra costs of biomarker measurement.

\section{REFERENCES}

1. Vulto A, Louwman M, Rodrigus P, et al. Referral rates and trends in radiotherapy as part of primary treatment of cancer in South Netherlands, 1988-2002. Radiother Oncol 2006;78: 131-137.

2. Ball D, Smith J, Wirth A, et al. Failure of T stage to predict survival in patients with non-small-cell lung cancer treated by radiotherapy with or without concomitant chemotherapy. Int $J$ Radiat Oncol Biol Phys 2002;54:1007-1013.

3. Bradley JD, Ieumwananonthachai N, Purdy JA, et al. Gross tumor volume, critical prognostic factor in patients treated with three-dimensional conformal radiation therapy for non-smallcell lung carcinoma. Int J Radiat Oncol Biol Phys 2002;52: 49-57.

4. Dehing-Oberije C, De Ruysscher D, van der Weide H, et al. Tumor volume combined with number of positive lymph node stations is a more important prognostic factor than TNM stage for survival of non-small-cell lung cancer patients treated with (chemo)radiotherapy. Int J Radiat Oncol Biol Phys 2008;70:10391044.

5. Brundage MD, Davies D, Mackillop WJ. Prognostic factors in non-small cell lung cancer: A decade of progress. Chest 2002; 122:1037-1057.

6. Pfister DG, Johnson DH, Azzoli CG, et al. American Society of Clinical Oncology treatment of unresectable non-small-cell lung cancer guideline: Update 2003. J Clin Oncol 2004;22: 330-353.

7. Firat S, Byhardt RW, Gore E. Comorbidity and Karnofksy performance score are independent prognostic factors in stage III non-small-cell lung cancer: An institutional analysis of patients treated on four RTOG studies. Radiation Therapy Oncology Group. Int J Radiat Oncol Biol Phys 2002;54:357-364.

8. Chemotherapy for non-small cell lung cancer. Non-small Cell Lung Cancer Collaborative Group. Cochrane Database Syst Rev 2000:CD002139.

9. Basaki K, Abe Y, Aoki M, et al. Prognostic factors for survival in stage III non-small-cell lung cancer treated with definitive radiation therapy: Impact of tumor volume. Int J Radiat Oncol Biol Phys 2006;64:449-454.

10. Werner-Wasik M, Swann RS, Bradley J, et al. Increasing tumor volume is predictive of poor overall and progression-free survival: Secondary analysis of the Radiation Therapy Oncology Group 93-11 Phase I-II radiation dose-escalation study in pa- tients with inoperable non-small-cell lung cancer. Int $J$ Radiat Oncol Biol Phys 2008;70:385-390.

11. Zhao L, West BT, Hayman JA, et al. High radiation dose may reduce the negative effect of large gross tumor volume in patients with medically inoperable early-stage non-small cell lung cancer. Int J Radiat Oncol Biol Phys 2007;68:103-110.

12. Solan MJ, Werner-Wasik M. Prognostic factors in non-small cell lung cancer. Semin Surg Oncol 2003;21:64-73.

13. Dehing-Oberije C, Yu S, De Ruysscher D, et al. Development and external validation of prognostic model for 2-year survival of non-small-cell lung cancer patients treated with chemoradiotherapy. Int J Radiat Oncol Biol Phys 2009;74:355-362.

14. Wulfkuhle J, Espina V, Liotta L, et al. Genomic and proteomic technologies for individualisation and improvement of cancer treatment. Eur J Cancer 2004;40:2623-2632.

15. Boldrini L, Donati V, Dell'Omodarme M, et al. Prognostic significance of osteopontin expression in early-stage non-smallcell lung cancer. Br J Cancer 2005;93:453-457.

16. Donati V, Boldrini L, Dell'Omodarme M, et al. Osteopontin expression and prognostic significance in non-small cell lung cancer. Clin Cancer Res 2005;11:6459-6465.

17. Kaminska J, Kowalska M, Kotowicz B, et al. Pretreatment serum levels of cytokines and cytokine receptors in patients with non-small cell lung cancer, and correlations with clinicopathological features and prognosis. M-CSF-An independent prognostic factor. Oncology 2006;70:115-125.

18. Koukourakis MI, Giatromanolaki A, Sivridis E, et al. Lactate dehydrogenase-5 (LDH-5) overexpression in non-small-cell lung cancer tissues is linked to tumour hypoxia, angiogenic factor production and poor prognosis. Br J Cancer 2003;89:877885 .

19. Le QT, Chen E, Salim A, et al. An evaluation of tumor oxygenation and gene expression in patients with early stage non-small cell lung cancers. Clin Cancer Res 2006;12:1507-1514.

20. Orditura M, De Vita F, Catalano G, et al. Elevated serum levels of interleukin-8 in advanced non-small cell lung cancer patients: Relationship with prognosis. J Interferon Cytokine Res 2002; 22:1129-1135.

21. Songur N, Kuru B, Kalkan F, et al. Serum interleukin-6 levels correlate with malnutrition and survival in patients with advanced non-small cell lung cancer. Tumori 2004;90:196200. 
22. Muley T, Dienemann H, Ebert W. CYFRA 21-1 and CEA are independent prognostic factors in 153 operated stage I NSCLC patients. Anticancer Res 2004;24:1953-1956.

23. Pujol JL, Molinier O, Ebert W, et al. CYFRA 21-1 is a prognostic determinant in non-small-cell lung cancer: Results of a metaanalysis in 2063 patients. Br J Cancer 2004;90:2097-2105.

24. Swinson DE, Jones JL, Richardson D, et al. Carbonic anhydrase IX expression, a novel surrogate marker of tumor hypoxia, is associated with a poor prognosis in non-small-cell lung cancer. $J$ Clin Oncol 2003;21:473-482.

25. De Ruysscher D, Wanders R, van Haren E, et al. HI-CHART: A phase I/II study on the feasibility of high-dose continuous hyperfractionated accelerated radiotherapy in patients with inoperable non-small-cell lung cancer. Int J Radiat Oncol Biol Phys 2008;71:132-138.

26. Maastricht Radiation Oncology, The Netherlands. Biobank Lung Cancer. Storing blood and protein of patients with lung cancer. NLM identifier: NCT00573040. ClinicalTrials.gov Web site. Bethesda, MD: National Library of Medicine. Available from: URL: http://clinicaltrials.gov/show/NCT00181519. Accessed January 11, 2010.

27. Senan S, De Ruysscher D, Giraud P, et al. Literature-based recommendations for treatment planning and execution in highdose radiotherapy for lung cancer. Radiother Oncol 2004;71: 139-146.

28. Maastricht Radiation Oncology, The Netherlands. Radiotherapy for NSCLC to an individualized MLD (BRONC MLD). NLM identifier: NCT00573040. ClinicalTrials.gov Web site. Bethesda, MD: National Library of Medicine. Available from: URL: http://clinicaltrials.gov/show/NCT00573040. Accessed December 28, 2009.

29. van Baardwijk A, Wanders S, Boersma L, et al. Mature results of an individualized radiation dose prescription study based on normal tissue constraints in stages I to III non-small-cell lung cancer. J Clin Oncol 2010;28:1380-1386.

30. Maastricht Radiation Oncology, The Netherlands. Concurrent chemo-radiation for NSCLC to an individualized MLD. NLM identifier: NCT00572325. ClinicalTrials.gov Web site. Bethesda, MD: National Library of Medicine. Available from:
URL: http://clinicaltrials.gov/show/NCT00572325. Accessed December 28, 2009

31. Cristianini N, Shaw-Taylor J. An introduction to support vector machines and other kernel-based learning methods. Cambridge: Cambridge University Press; 2000.

32. Platt JC. Probabilities for SV machines. In: Smola AJ, Bartlett P, Scholkopf B, editors. Advances in large margin classifiers. Cambridge: MIT Press; 2000.

33. Hammarstrom $\mathrm{S}$. The carcinoembryonic antigen (CEA) family: Structures, suggested functions and expression in normal and malignant tissues. Semin Cancer Biol 1999;9:67-81.

34. Ballesta AM, Molina R, Filella X, et al. Carcinoembryonic antigen in staging and follow-up of patients with solid tumors. $T u$ mour Biol 1995; 16:32-41.

35. Nieder C, Nestle U, Ukena D, et al. Tumor markers as prognostic factors in non-small-cell bronchial carcinoma. Strahlenther Onkol 1995;171:587-593.

36. Coussens LM, Werb Z. Inflammation and cancer. Nature 2002; 420:860-867.

37. Krysan K, Lee JM, Dohadwala M, et al. Inflammation, epithelial to mesenchymal transition, and epidermal growth factor receptor tyrosine kinase inhibitor resistance. J Thorac Oncol 2008;3:107-110.

38. Rollins BJ. Inflammatory chemokines in cancer growth and progression. Eur J Cancer 2006;42:760-767.

39. Sin DD, Man SF. Systemic inflammation and mortality in chronic obstructive pulmonary disease. Can J Physiol Pharmacol 2007;85:141-147.

40. Cox JD, Pajak TF, Asbell S, et al. Interruptions of high-dose radiation therapy decrease long-term survival of favorable patients with unresectable non-small cell carcinoma of the lung: Analysis of 1244 cases from 3 Radiation Therapy Oncology Group (RTOG) trials. Int J Radiat Oncol Biol Phys 1993;27: 493-498.

41. Goldstraw P, Crowley J, Chansky K, et al. The IASLC Lung Cancer Staging Project: Proposals for the revision of the TNM stage groupings in the forthcoming (seventh) edition of the TNM Classification of malignant tumours. J Thorac Oncol 2007;2:706-714. 